\title{
Numerical prediction research on the evaporation rate of liquid cargo tank for LNG Filling Vessel Based on Ansys
}

\author{
WANG Yunlong ${ }^{*}$ YUN Yanyun, CHEN Ming, LIN Yan \\ School of Naval Architecture, Dalian University of Technology, Dalian, China, 116024 \\ Email: yunlongw@dlut.edu.cn; yuyanyun@dlut.edu.cn
}

\begin{abstract}
Key words: LNG Filling Vessel; Finite Element Analysis; Cargo Containment System; Evaporation Rate; Numerical Prediction

Abstract: With the increase of LNG power ship, the facility and equipment of LNG fuel filling for LNG power ship have become an important problem. LNG filling vessel is a good development direction of LNG filling technology because of its advantages of self navigation ability, safety and reliability, flexible scheduling. According to the characteristics of LNG filling vessel cargo containment system, this paper explores the evaporation rate numerical prediction method under different loading conditions of liquid cargo tank, it can make quick calculations on the steady-state evaporation rate of different insulation thickness, and gets the corresponding thickness value of thermal insulation layer to guide the LNG filling vessel design. Through the research, it can be found that average daily evaporation rate of the $C$ type cargo containment system declines with the declining of liquid level. The research results of this paper can provide technical support for the design of $\mathrm{C}$ type cargo containment system.
\end{abstract}

\section{Introduction}

With the increase of LNG power ship, the facility and equipment of LNG fuel filling for LNG power ship have become an important problem in shipping industry. LNG filling ship is an good development direction of LNG filling technology because of its advantages of self navigation ability, safety and reliability, flexible scheduling[1]. At present, all the shipbuilding countries are actively carrying out the research and development of technical reserves for LNG filling ship[2]. The main difference between the LNG filling ship and the LNG transport ship is that most moment of the LNG filling ships are not full and not empty during the voyage. It is necessary to consider the effect of free surface and cargo sloshing on ship stability and structure, and the prediction and control of evaporation rate under different load rate conditions for LNG filling ship. Most of the international existing LNG filling ships and concept ships use the $C$ type Cargo containment system. Based on the Ansys finite element method, the researches of the numerical prediction method for evaporation rate of the $\mathrm{C}$ type cargo containment system of LNG filling ship will provide technical support for the design of LNG filling ship.

\section{Heat transfer analysis method based on Ansys software}

According to the energy conservation principle of the thermal module in Ansys software, the heat balance equation is obtained. At the same time, the finite element method is used to analyze and calculate the temperature field distribution, the increase or consume of heat for each node. Three kinds of transmission modes are considered during the thermodynamic treatment of Ansys: heat conduction, heat convection and heat radiation. The liquid cargo tank of the medium and small LNG vessel is the horizontal pressure vessel, the liquid hold of cargo containment system are 
galvanized thin steel plate, thermal insulation layer, liquid hold inner shell from outside to inside[3]. The heat transfer model of liquid cargo tank full filled of LNG filling ship is shown in Figure 1.

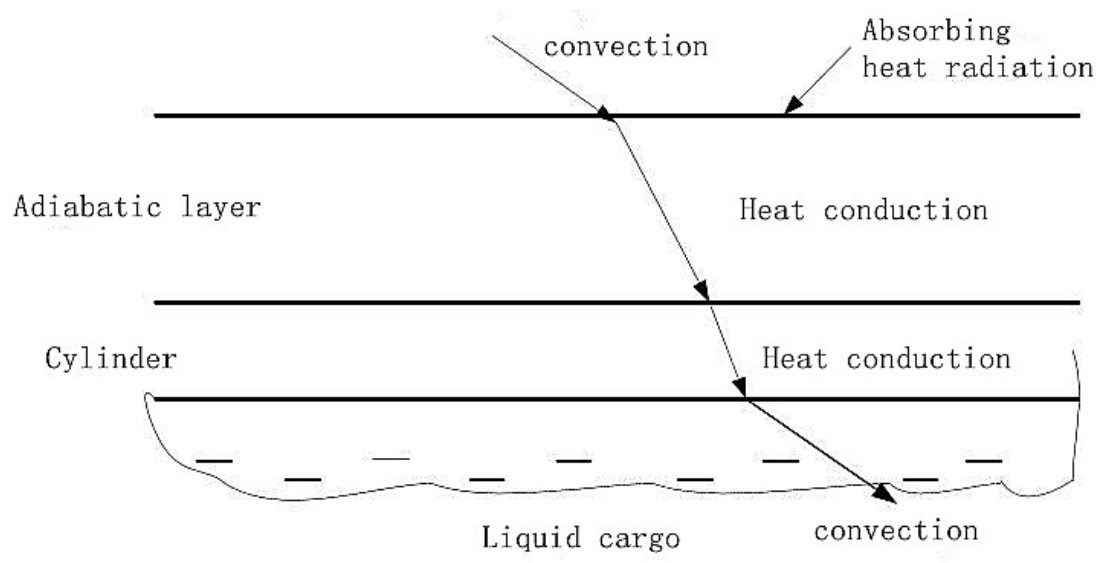

Fig.1 Schematic diagram of the heat transfer model of liquid cargo tank full filled with LNG

Because the coefficient of heat convection is difficult to determine[4,5], the simplest algorithm is to apply the temperature inside and outside the thermal insulation layer to form a fixed boundary condition in the heat analysis of the $\mathrm{C}$ tank thermal of the LNG filling vessel[6]. The outside of the insulation layer always keeps the outside temperature, and the inner side always keeps the boiling point temperature of the liquid. Put the resulting leakage of heat into the formula to calculate the rate of evaporation. The calculation formula of evaporation rate is as follows.

$$
B O R=\frac{\text { heat } \times 3600 \times 24}{\text { dens } \times \text { lamta } \times v} \times 100 \%
$$

where heat is leakage of heat, $\mathrm{kW} ; B O R$ is daily evaporation rate, $\mathrm{kg}$; dens is density of LNG, $\mathrm{kg} / \mathrm{m} 3$; lamta is latent heat of evaporation, $\mathrm{kJ} / \mathrm{kg}$; $v$ is total loading volume of LNG liquid tank, $\mathrm{m}^{3}$.

The following formula is obtained by using the previous research on the heat convection in the heat analysis of the $\mathrm{C}$ tank thermal of the LNG filling vessel.

$$
\alpha=1.163\left(2+10 \sqrt{V_{S}}\right)
$$

Where $\alpha$ is heat convection coefficient of fluid with respect to the heat dissipation surface, $\mathrm{W} / \mathrm{m}^{2} \cdot \mathrm{K} ; V_{S}$ is service speed of $\mathrm{LNG}$ Vessel, $\mathrm{kn}$.

\section{The numerical simulation and the rate of evaporation forecast of fully loaded liquid cargo tank of LNG filling vessel}

Taking the temperature field of the liquid cargo tank of the $1000 \mathrm{~m}^{3} \mathrm{LNG}$ filling ship as an example, the numerical prediction method of the evaporation rate of the independent $\mathrm{C}$ type liquid hold is explored. The liquid cargo tank loaded is LNG which temperature is $-163^{\circ} \mathrm{C}$, we choose the steel that contain $9 \%$ nickel as the inner material of the liquid cargo tank, urethane foam material with excellent thermal insulation effect as thermal insulation layer, and the outermost layer of cargo tank is zinc-coated steel sheet to ensure the cryogenic carrying environment of liquid cargo tank when operating. The daily evaporation rate of LNG which is in cargo tank is not more than 3\%. The preliminary design related parameters of $1000 \mathrm{~m}^{3}$ LNG filling vessel's liquid cargo tank are as shown in Table 1. We predict whether the evaporation rate could meet control requirement when 
thermal insulating layer thickness is $300 \mathrm{~mm}$ by FEM analysis.

Table 1 The preliminary design related parameters of $1000 \mathrm{~m}^{3}$

LNG filling vessel's liquid cargo tank

\begin{tabular}{ll}
\hline Items & Numerical value \\
\hline The total length of tanks & $33.4 \mathrm{~m}$ \\
The end socket's diameter & $6.4 \mathrm{~m}$ \\
The position of saddle & $\pm 9.6 \mathrm{~m}$ \\
The thickness of saddle & $0.3 \mathrm{~m}$ \\
Speed & $12 \mathrm{kn}$ \\
Computing environment temperature & $45^{\circ} \mathrm{C}$ \\
The heat conductivity of insulating layer & $0.022 \mathrm{~W} /(\mathrm{m} \cdot \mathrm{K})$ \\
The material of insulating layer & Urethane foam \\
The thickness of insulating layer & $300 \mathrm{~mm}$ \\
The install method of insulating layer & Foaming technique \\
The heat conductivity of saddle & $0.15 \mathrm{~W} /(\mathrm{m} \cdot \mathrm{K})$ \\
\hline
\end{tabular}

In the numerical simulation of the heat transfer of the liquid hold, the following assumptions are made:

(1) The thickness of thermal insulation layer is equally distributed, and ignore the effect of transformation of local thickness when analysis;

(2) The temperature of the external environment is not change with the change of time and place in a certain working condition;

(3) The materials of cargo tank's inner shell and insulation layer are homogeneity and isotropy, the thermal conductivity of each material is not changed due to the change of the temperature.

Based on Ansys software, the numerical prediction procedure of the evaporation rate for the liquid cargo of LNG filling vessel is as follows:

(1) In accordance with the actual size to make numerical modeling and meshing for cargo tank, thermal insulating layer, saddle and piping by using Ansys software. It using four-node shell element SHELL181 for the web plate structure of cargo tank and saddle, using two-node beam element BEAM188 for the bezel panel structure of saddle, and using eight- node solid element SOLID45 for hardwood. The side of mesh is $\mathrm{R} / 15$ ( $\mathrm{R}$ is the radius of liquid cargo tank ), it is $200 \mathrm{~mm}$, the type of mesh is quadrilateral, the sum of nodes are 24132, and the sum of elements are 23064, all of it shown in Figure 2 and Figure 3.

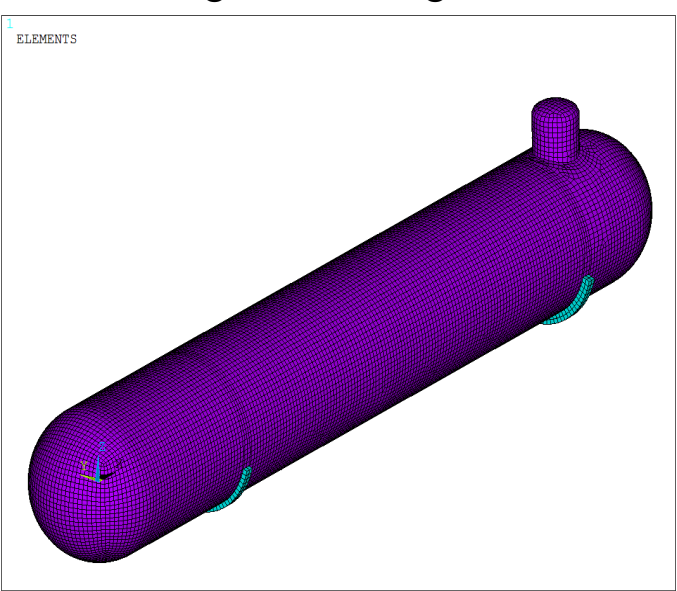

Fig. 2 Meshing of liquid cargo tank-overall

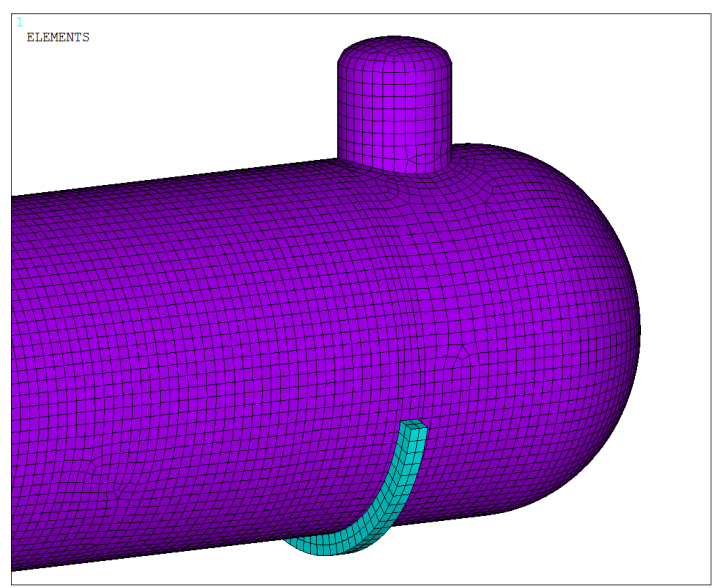

Fig.3 Meshing of liquid cargo tank-air chamber and saddle 
(2) According to the actual physical properties gives physical properties to the numerical model.

(3) To give loaded liquid stable liquid temperature in the inside Insulation layer as boundary conditions, and to give coefficient of heat convection according to heat convection in the outer Insulation layer.

(4) Calculating the maximum rate of evaporation when full loaded.

Under the condition of the corresponding table 1, it can obtain the daily evaporation rate was $3.340 \%$, and it is not satisfied the design requirements by calculation.

Changing the thickness of thermal insulating layer, it shows that the daily evaporation rate could be controlled at $2.97 \%$ when its thickness is $340 \mathrm{~mm}$. it is satisfied the design requirements when the thickness is $340 \mathrm{~mm}$.

\section{LNG filling vessel's prediction of evaporation rate under different loading rate}

After the filling operation, the majority of the LNG filling vessel is not full, and the non empty cabin, the prediction and control of the evaporation rate under different load conditions are needed.

As the main component of LNG is methane and nitrogen, related technical manuals show that the thermal conductivity of methane and nitrogen are almost equal at the ambient condition of $100 \mathrm{~K}$ to $150 \mathrm{~K}$, so it can be assumed that LNG gas has single thermal conductivity. Based on steady-state temperature field theory, it's can suppose that the liquid portion has a stable liquid temperature, so fixed boundary conditions are used. Gas is modelled in the form of steady-state temperature field, and assigned one equivalent thermal conductivity parameter, at the junction with the liquid portion still use the fixed boundary conditions. Such an algorithm can be more close to the real situation, and specific gas medium thermal conductivity can be determined by comparing the numerical results and experimental measurement data. The advantage of this algorithm is that the evaporation rate under different loading rates has a more accurate estimate, and it can predict the values of evaporation rate under different loading rate of liquid cargo tanks. Through experimental verification, the value of gas phase thermal conductivity can be $0.2 \mathrm{~W} /(\mathrm{m} \cdot \mathrm{K})$, the gas temperature field distribution of 1000m3 LNG filling vessel is showed in Figure 4.

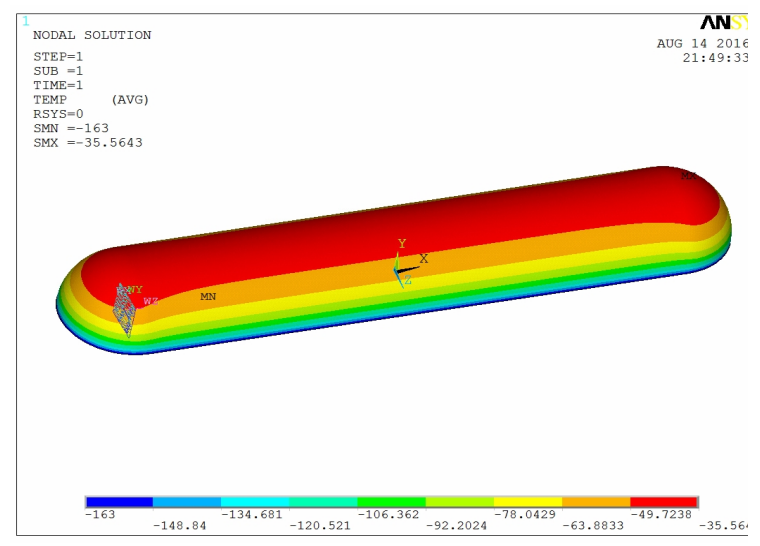

Fig.4 Gas temperature field of $1000 \mathrm{~m}^{3} \mathrm{LNG}$ tank

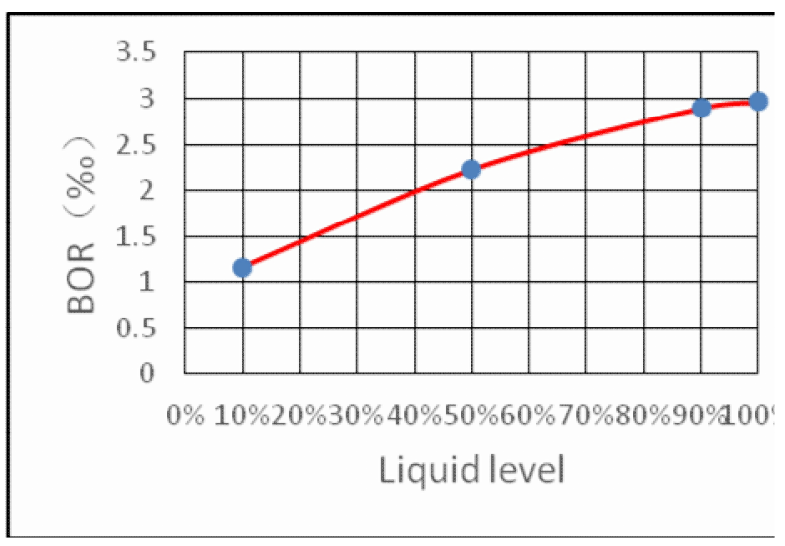

Fig.5 The relationship between evaporation rate and liquid level of $1000 \mathrm{~m}^{3}$ tank

According to the above theories and hypothesises, it can be calculated that the average daily evaporation rate is $2.233 \%$ when the thickness of insulation layer is $340 \mathrm{~mm}$ and loading rate is $50 \%$. Simultaneously, calculating the evaporation rate under different liquid level and drawing the curve of daily evaporation rate with the liquid level changing, and it's showed in Figure 5. 


\section{Summary}

The cargo support system is the most critical core technology of LNG ship, the optimal design of thermal insulation layer and the accurate prediction and effective control of evaporation rate are the research hotspot and difficulty in recent years.According to the characteristics of LNG filling vessel cargo containment system, this paper puts forward a numerical prediction method of the evaporation rate for liquid cargo tank under different loading conditions, it can make quick calculation on the steady-state evaporation rate of different thermal insulation thickness, and to guide the design. Through the study can found the $\mathrm{C}$ type cargo containment system's average daily evaporation rate declines with the declining of liquid level. The research results of this article can provide technical support for the design of $\mathrm{C}$ type cargo containment system.

\section{Acknowledgments}

Sponsored by the National Natural Science Foundation of China (Grant NO. 51209034); Natural Science Foundation of Liao Ning Province (Grant No. 2014020017).

\section{References}

[1] American Bureau of Shipping. Bunkering of Liquefied Natural Gas-fueled Marine Vessels in North America. Washington DC, American Bureau of Shipping . 2013.

[2] Xu Jianyong, Fan Hongjun, Wu Shunping. Research on LNG Ship to Ship (STS) Bunkering Operations, J.Ship Engineering. 2015,37(1): 7-10.

[3] Yu Xiang hu. Research on the LNG carrier tank's thermal transfer and stress finite element analysis. Wuhan University of Technology, 2009.

[4] Miana M, del Hoyo R, Rodrigálvarez V, Valdés J R, Llorens R. Calculation models for prediction of liquefied natural gas(LNG) ageing during ship transportation, J. Applied Energy. 2010, 87(5):1687-1700.

[5] Jang Hyun Lee, Yoon Jo Kim. Computational study of LNG evaporation and heat diffusion through a LNG cargo tank membrane, J. Ocean Engineering. 2015, 106(15):77-86.

[6] Yang Bo, Sheng Sujian. On General Arrangement Design of 1000 m3 LNG Fuel Bunker Vessel, J.Ship and Ocean Engineering. 2014, 43(5): 138-141. 